\title{
New Methods
}

\author{
Roland W. Pfäffle \\ University Children's Hospital, Leipzig, Germany
}

\section{Nanobiotechnology}

Nanotechnology has developed over the past 15 years slowly and steadily in the hands of engineerscientists. However, it recently has raised more and more attention in medical research as devices were developed that hold promise to facilitate and improve diagnostic procedures even before these technologies will have their impact on therapeutic approaches. As nano-objects hold promise to facilitate multiplexing of hormone measurements and cut down on sample volumes even further, these techniques will be of special interest for the paediatric endocrinologist. Next to these improvements, such techniques have the potential to facilitate serial hormone measurements in vivo, promising something that would function as an artificial hormonal gland. Two papers are introduced that give an interesting outlook on the recent developments as well as a prospective risk assessment of a technology that is just starting to enter our scientific horizon.

\section{Nanobiotechnology: the promise and reality of new approaches to molecular recognition}

Fortina P, Kricka LJ, Surrey S, Grodzinski P

Center for Translational Medicine, Department of Medicine, Thomas Jefferson University, Philadelphia, Pa., USA

paolo.fortina@jefferson.edu

Trends Biotechnol 2005;23:168

Background: The authors define nanobiotechnology as the convergence of engineering and molecular biology that is leading to a new class of multifunctional devices and systems for biological and chemical analysis with better sensitivity and specificity and a higher rate of recognition. Nano-objects with important analytical applications include nanotubes, nanochannels, nanoparticles, nanopores and nanocapacitors.

Methods: The authors take a critical look at a subset of recent developments in the area relevant to recognition of molecules. They describe the potential benefits of using nano-objects like nanotubes, quantum dots, nanorods and nanoprisms as well as nanodevices like nanocapacitors, nanopores and nanocantilevers. The potentials that lead to expanded ranges of label multiplexing are described along with potential applications in future diagnostics.

Conclusions: The authors speculate on future pathways in nanotechnology development and they conclude that the time has come for clinical application of these developments from this somewhat chaotic, yet promising, new field.

This is a comprehensive overview of the various devices that have been developed in the area of nanotechnology. The authors put an emphasis on the devices that have their applications in nanobiotechnolgy. Despite common anticipation about this new area, the applications that are likely to enter clinical medicine soon affect diagnostic procedures rather than therapeutic strategies. Many of the techniques affected, like multiplexing labels for serial hormone measurements, will be of primary interest to the clinical chemist. However these techniques also hold promise to enable the design of new diagnostic strategies for hormonal disorders which is one reason why the paediatric endocrinologist should be aware of them even in this very early stage of its development. 


\section{The case of nanobiotechnology}

Grunwald A

Institute of Technology Assessment and Systems Analysis (ITAS) at the Research Centre Karlsruhe, Germany

armin.grunwald@itas.fzk.de

EMBO Rep 2004;5(Spec No S32)

Background: Nanobiotechnology has raised considerable scientific, political and public interest. The possibility of controlling matter at the molecular and atomic level for further miniaturization of components, products and methods is fascinating and presents far-reaching possibilities for application. A differentiation is made into so-called 'nano2bio' technologies, which use nanotechnology for the analysis and production of biological nanosystems, and 'bio2nano' techniques, which use biotic materials and structures to build technical nanosystems. The first would be the technique to affect clinical medicine first. The potential of this technique is so remarkable that ethical reflection seems almost superfluous, however every new method holds risks which may cause irreversible damage.

Methods: The author discusses the different public and scientific standpoints that exist on the new evolving technique of nanobiotechnology. He gives examples on former developments in science and technology where an inadequate risk assessment had led to an early and extensive use of a new technology with the result of unanticipated hazards to the individual and the public, which finally has impaired or at least delayed further developments of these techniques. The author places the potential risk of a new technology into four main categories: risk of accidents; risk to health; risk to the environment; and social risks. He discusses the foreseeable aspects of this new technology in this respect.

Conclusion: The author argues that an early open discussion of possible technological risks contributes, in the medium and long term, towards upholding and strengthening confidence in this research, science and technology. The further development of nanobiotechnology therefore has to be accompanied by continuous research on possible risks and its ethical and political implications. A so-called 'vision assessment' would have to investigate positive and negative utopias and involve an open dialogue with the participation of nanobiotechnologists, the medical profession and people specialized in technology assessment.

This is an interesting essay, not only about the possible risk-benefit ratio of the new field of nanobiotechnolgy. Rather, it is a contemplative approach to the possible ways how any new evolving technology could be assessed in respect to their potentials and possible risks and how this should be communicated to the scientific community and the public in general. In this article it also becomes clear that the feared risks of nanobiotechnology have little to do with the foreseeable applications such as those described in the above-mentioned article. They rather concern its 'visionary' and still speculative applications. This article is not meant to introduce the reader to various techniques involved in nanobiotechnology, but it helps the scientist to understand that the introduction of this technology is still an open process which holds the possibility that one can avoid many of the mistakes that have been made with new technologies in the past.

\section{Nanoparticle labels in immunosensing using optical detection methods}

\section{Seydack M}

8sens.biognostic AG, Nanobiotechnology Group, Berlin, Germany

seydack@biognostic.de

Biosens Bioelectron 2005;20:2454

Background: Efforts to improve the performance of immunoassays and immunosensors by incorporating different kinds of nanostructures have gained considerable momentum over the last decade. Apart from liposomes, not discussed here, most groups focus on artificial, particulate marker systems, both organic and inorganic.

Methods: The underlying detection procedures used by nanobiotechnological approaches may be based either on electromagnetic or optical techniques. In this review the authors give an outlook mainly on the optical techniques generating signals as diverse as static and time-resolved luminescence, one- and two-photon absorption, Raman and Rayleigh scattering as well as surface plasmon resonance and others. All techniques described are geared to achieve one or more of the following goals for modern diagnostic procedures: lowering of detection limits (even as low as the so-called single-molecule level), 
parallel integration of multiple signals (multiplexing), signal amplification by several orders of magnitude and prevention of photobleaching effects with concomitant maintenance of antigen binding specificity and sensitivity.

Conclusions: Inorganic nanoparticle labels based on noble metals, semiconductor quantum dots and nanoshells appear to be the most versatile systems for these bioanalytical applications.

This review article may not be accessible (and understandable) right away for many clinical scientists, but it gives a good overview and an introduction into the applications of nanobiotechnology that will be most readily availably for medical research. These techniques involve almost entirely diagnostic applications that should have significant effects on future research in paediatric endocrinology.

\section{New imaging techniques}

Molecular genetics have come to a state where it becomes increasingly important to study the regulation of genes and gene families in the context of the cellular and nuclear structures. Past experiments have focused on identification of the genes and proteins involved in gene transcription and intracellular signaling. However, not all effects of the regulating factors become evident from their interactions with each other or with certain parts of the genome. Rather, some of the functional properties directly relate to how these factors are distributed within the various cell compartments and how they migrate between them as a consequence of specific stimulatory effects. Some of the pathophysiological consequences of mutations within factors of a signaling cascade are possibly more influenced by the consequences of these mutations on their intracellular localization than by the effects on protein-protein or protein-DNA interaction. Many of the interpretations deduced from in vitro assays may therefore appear under a new light, when seen in the context of such analyses. The two new technologies that hold high promises in studying processes that regulate genes down to the promoter level accurately are the chromatin immunoprecipitation (ChIP)-based assays and the so-called live single cell studies using photobleaching techniques.

\section{Imaging molecular interactions in living cells}

Day RN, Schaufele F

Department of Medicine, University of Virginia Health Sciences Center, Charlottesville, Va., USA

rnd2v@virginia.edu

Mol Endocrinol 2005; 19:1675

Background: This review addresses the question how hormones integrate the activities of their target cells through receptor-modulated cascades of protein interactions that ultimately lead to changes in cellular function. Understanding how the cell assembles these signaling protein complexes is critically important to unraveling disease processes, and to the design of therapeutic strategies.

Methods: The authors demonstrate how recent advances in live-cell imaging technologies, combined with the use of genetically encoded fluorescent proteins, allow the assembly of these signaling protein complexes to be tracked within the organized microenvironment of the living cell. They review some of the recent developments in the application of imaging techniques to measure the dynamic behavior of factors of a signaling cascade or transcription factors in the cell nucleus. In addition, they describe how these techniques allow the detection of colocalization of these factors in specific cell compartments, and how it becomes possible with these techniques to assess the spatial relationships between proteins in living cells.

Results: It becomes clear that changes of hormone levels alter nuclear receptor localization, mobility, and interactions in different subcellular compartments. The authors also discuss different means of measurements that define the spatial relationships and dynamics between proteins in living cells. These techniques include fluorescence colocalization, fluorescence recovery after photobleaching, fluorescence correlation spectroscopy, fluorescence resonance energy transfer microscopy, and fluorescence lifetime imaging microscopy. 
Conclusions: The techniques discussed in this review show that live-cell imaging tools provide an important complement to biochemical and structural biology studies, extending the analysis of protein-protein interactions, protein conformational changes, and the behavior of signaling molecules to their natural environment within the intact cell.

This is a comprehensive review about so-called live-cell imaging techniques such as fluorescence colocalization studies, fluorescence recovery after photobleaching (FRAP) and fluorescence resonance energy transfer microscopy. It is an excellent introduction to the different aspects of each of these approaches as it describes their advantages and limitations in interpreting the data obtained with each of the methods. It becomes clear that only a combination of these will provide a perspective as close to reality as possible. On the other hand it also becomes evident that all of these applications are technically quite demanding and may not be readily accessible to many research laboratories. However, understanding these methods will help any individual investigator to adequately decide which approach to take in complementing his or her biochemical findings when dealing with cellular signaling processes.

\section{Molecular dynamics and nuclear receptor function}

Hinojos CA, Sharp ZD, Mancini MA

Department of Molecular and Cellular Biology, Baylor College of Medicine, Houston, Tex., USA

Trends Endocrinol Metab 2005;16:12

Background: The development of live cell and biochemical analysis methods has led to an increase in our understanding of the dynamic regulation of transcription. Live single cell studies using photobleaching techniques indicate that many proteins have a high nuclear mobility. Pioneering work using promoter array systems based on the lac operon or the mouse mammary tumor virus promoter enabled the study of chromatin structure, promoter occupancy and protein-chromatin interaction dynamics in relation to transcription. Chromatin immunoprecipitation (ChIP)-based assays allow an exhaustive analysis of the temporal recruitment of proteins to an endogenous promoter and provide evidence of cyclic proteinprotein and protein-promoter interactions.

Methods: The evolution of the technology of fluorescence recovery after photobleaching is described chronologically starting with the construction of a hamster ovary cell line with stably integrated repeats of the lac operator. This initial system was geared towards studies of nuclear and chromosomal ultrastructures in response to various stimulatory signals. Although this is far from a physiological surrounding of a specific hormone-producing cell, it gave insight into the reorganization of chromatin in response to transcriptional activation. It led to a new concept of highly dynamic pattern of receptorcoregulator complexes that control gene transcription and to the suggestion that the rapid movement of many nuclear proteins might minimize the need for preformed or long-lived stable protein complexes. The recent advances in the refinement of this system are described as long with the potential new applications. In contrast to the techniques of living cell microscopy, chromatin immunoprecipitation (ChIP) studies involve a different approach: tissue or cells are cross-linked with formaldehyde and chromatin fragmented, thereafter chromatin fragments are immunoprecipitated with antibodies against specific nuclear regulators or coregulators and after reversing the cross-linking the promoter DNA fragments are detected. This approach has proven increasingly useful in situations where nuclear receptors have multiple nuclear targets. Recent developments include adaptations of this technique to allow kinetic studies over time or in response to hormone stimulation. Among the nuclear receptors studied so far more extensively is estrogen receptor (ER).

Conclusions: Although reflecting different timescales, both ChIP and live cell studies indicate a highly dynamic control of transcription that until now has gone undetected and unappreciated. Which of the two approaches is the one more closely representing reality remains somewhat questionable as both methods obviously carry their limitations. The authors speculate that the use of faster, less phototoxic approaches (e.g. multiphoton FRAP imaging) for live cell imaging and the development of faster crosslinking methods (e.g. UV laser cross-linking) could bridge the gap that still exists and thereby would help to gain a comprehensive view of the processes involved in gene regulation.

This paper focuses on the dynamics of nuclear receptors and coregulators. The two main approaches discussed are the techniques of live cell imaging (like FRAP) and chromatin immunoprecipitation 
(ChIP). Although the technicalities of each of the methods are not described in detail, this overview gives a comprehensive insight into the historical development of the techniques, their past and current applications as well as their advantages and limitations under certain conditions. It becomes clear that these methods will be indispensable for many researchers examining the effects of hormones on the cellular and nuclear level although they are not yet in general use. With continued advances in both live cell and biochemical approaches and with improved cellular models, the molecular research on hormonal cell system will however soon have a broader access to these fascinating new techniques.

\title{
Inactivating Pit-1 mutations alter subnuclear dynamics suggesting a protein misfolding and nuclear stress response
}

\author{
Sharp ZD, Stenoien DL, Mancini MG, Ouspenski II, Mancini MA \\ Department of Molecular and Cellular Biology, Baylor College of Medicine, Houston, Tex., USA \\ J Cell Biochem 2004;92:664
}

Background: Pit-1 (POU1F1) is a POU-class nuclear DNA-binding transcription factor which specifies three of the parenchymal cell types in anterior pituitary ontogeny.

Methods: The authors used fluorescent fusions and live cell imaging and compared the dynamic behavior of wild-type and inactivating Pit-1 point mutations.

Results: Fluorescence recovery after photobleaching (FRAP) and real-time extraction data indicated that wild-type Pit- 1 has a dynamic mobility profile, with $\mathrm{t}_{1 / 2} \mathrm{~s}$ approximately $5-7 \mathrm{~s}$ when expressed from low to high amounts, respectively. The authors found that according to direct observations during extraction, Pit-1 is retained to approximately $50 \%$, indicating a dynamic interaction with the nucleosome. When the authors expressed Pit-1 carrying two different debilitating mutations transiently they could find the mutants to translocate normally to the cell nucleus, however they found two different levels of mobility, which both were clearly distinguishable from wild-type Pit-1. Whereas at low expression levels, the $t_{1 / 2} \mathrm{~s}$ of Pit(W261C) and Pit(A158P) mutants are extremely rapid (0.3 and $0.6 \mathrm{~s} \mathrm{t} \mathrm{t}_{1 / 2} \mathrm{~s}$, respectively). They both become immobilized and insoluble at higher expression levels, unlike wild-type Pit-1. The mutants then show complete cofractionation with the insoluble nuclear matrix. The decreased mobility of Pit(A158P) relative to Pit(W261C) at low expression levels correlates with its ability to partially activate when expressed at low levels and its ability to bind cognate DNA. At high expression levels, lower Pit(A158P) activation correlates with its immobilization and insolubility.

Conclusion: The authors deduce from their data that a link exists between specific rates of intranuclear mobility and Pit- 1 transcription function. They assume that their data imply inactivating mutations can lead to an intranuclear sorting away from the transcription-related pathways, and at least in part to a misfolding of the protein. They conclude that caution is suggested when interpreting point (or other) mutational analyses of transactivator function, as new compartmentation, especially in the context of expression levels, may cloud the distinction between defining functional molecular domains and intranuclear processing of misfolded proteins.

This is a very nice example on how the dynamic studies obtained by live cell microscopy can at least shake some of the 'old' paradigms obtained by functional analyses alone. This study demonstrates the technique of fluorescent recovery after photobleaching (FRAP) on nuclear transcription factors like Pit-1. The authors give a detailed description of all the techniques involved. Their paper holds impressing pictures of Pit- 1 as it moves to the cell nucleus and forms a very specific pattern of intranuclear distribution, which is abrogated by the mutations that have been previously reported in humans with multiple pituitary hormone deficiency (MPHD). So they were able to explain the confusing finding why one of the mutations (A158P) is able to confer transactivation at a low level of transcription that however is inversely correlated to expression levels. The results of the FRAP images support the idea that at higher levels of expression this mutant protein becomes insoluble and ultimately less active. Overall this paper demonstrates that functional data using DNA-binding experiments and cotransfection assays only give a partial picture of the functional capabilities of a nuclear transcription factor and that live cellular imaging studies can effectively complement such data leading to new conclusions. 


\section{Rapid glucocorticoid receptor exchange at a promoter is coupled to transcription and regulated by chaperones and proteasomes}

Stavreva DA, Muller WG, Hager GL, Smith CL, McNally JG

Laboratory of Receptor Biology and Gene Expression, Center for Cancer Research,

National Cancer Institute and Light Imaging Facility, National Institute for Neurological Disorders and Stroke, Bethesda,

Md., USA

Mol Cell Biol 2004;24:2682-2697

Background: There is a fast exchange of the glucocorticoid receptor (GR) at promoter target sites. This system is the only known system in which transcription factor cycling at a promoter is so fast, occurring on a time scale of only seconds. The mechanism and function of this rapid exchange are unknown.

Methods: The authors use a tumor cell line under different experimental conditions (i.e. dexamethasone treatment) to perform studies on GR-responsive promoter.

Results: They provide evidence for the necessity of a proteasome activity for this rapid GR exchange at the promoter. They also show that chaperones, specifically hsp90, stabilize the binding of GR to the promoter, complicating models in which the associated chaperone, $\mathrm{p} 23$, has been proposed to induce GR removal.

Conclusions: The results are the first evidence to connect chaperone and proteasome functions in setting the residence time of a transcription factor at a target promoter. Moreover, the results demonstrate that longer GR residence times are consistently associated with greater transcriptional output, suggesting a new paradigm in which the rate of rapid exchange provides a means to tune transcriptional levels.

This example shows that not only the presence of various components of a transcriptional activator are determining the level of transcription, but also the turnover rate of various constituents influence the activity significantly. As the turnover rate of the glucocorticoid receptor is so high, this system seems ideally suited for a technique like fluorescent recovery after photobleaching (FRAP). This may however not be the case for other promoter systems with slower turnover rates. As the turnover rate of glucocorticoid receptor is influenced by factors that are not directly a part of the transactivation complex, this is a good example that dynamic studies can give a hint towards the mechanisms that regulate the cellular response to a signal like a hormone. Classical functional analysis would have missed this phenomenon which might carry important consequences also for therapeutic approaches.

\section{A serine/threonine-rich motif is one of three nuclear localization signals that determine unidirectional transport of the mineralocorticoid receptor to the nucleus}

Walther RF, Atlas E, Carrigan A, Rouleau Y, Edgecombe A, Visentin L, Lamprecht C, Addicks GC, Hache RJ, Lefebvre YA

Department of Medicine and Biochemistry, Graduate Program in Biochemistry, Ottawa Health Research Institute, Ottawa Hospital, University of Ottawa, Ont., Canada

J Biol Chem 2005;280:17549

Background: The mineralocorticoid receptor is a tightly regulated nuclear hormone receptor that transmits corticosteroid signals in a highly selective way. Steroid treatment transforms mineralocorticoid receptor from a transcriptionally inert state, in which it is distributed equally between the nucleus and cytoplasm, to an active completely nuclear transcription factor.

Methods: The authors report the characteristics of this receptor by studying its behavior using live cell microscopy and fluorescent recovery after photobleaching after introduction of various amino acid substitutions into the recombinant receptor.

Results: The authors show that mineralocorticoid receptor is an atypical nuclear hormone receptor that moves unidirectionally from the cytoplasm to the nucleus. They also demonstrate that nuclear import of mineralocorticoid receptor is controlled through three nuclear localization signals (NLS) of distinct types. Nuclear localization of naive mineralocorticoid receptor was mediated primarily through a novel serine/threonine-rich NLS (NL0) in the receptor N-terminus. Specific amino acid substitutions that mimicked phosphorylation selectively enhanced or repressed NLO activity, which highlighted the potential for active regulation of this new type of NLS. The second NLS (NL2) within the ligand-binding 
domain also lacks a recognizable basic motif. Nuclear transfer through this signal was strictly dependent on steroid agonist, but was independent of the interaction of mineralocorticoid receptor with coactivator proteins. The third mineralocorticoid receptor NLS (NL1) is a bipartite basic motif localized to the C-terminus of the mineralocorticoid receptor DNA-binding domain with properties distinct from those of NL1 of the closely related glucocorticoid receptor. NL1 acted in concert with NL0 and NL2 to stimulate nuclear uptake of the agonist-treated receptor, but also directed the complete nuclear localization of mineralocorticoid receptor in response to treatment with steroid antagonist.

Conclusion: The authors conclude from their results that the mineralocoid receptor presents a nuclear hormone receptor whose unidirectional transfer to the nucleus may be regulated through multiple intracellular pathways.

This example demonstrates that this new imaging technique enhances our insights of signals regulating gene promoters and allows the determination of the components necessary to enhance gene transcription. In this paper a comprehensive microdissection of the mineralocorticoid receptor is given by delineating the various domains within the receptor that influence the intracellular transportation of this molecule which may be one of the key mechanism of its physiological regulation.

\title{
Leptin signaling targets the thyrotropin-releasing hormone gene promoter in vivo
}

\author{
Guo F, Bakal K, Minokoshi Y, Hollenberg AN \\ Thyroid Unit, Division of Endocrinology, Beth Israel Deaconess Medical Center, Boston Mass., USA \\ Endocrinology 2004;145:2221
}

Background: The authors point out that the regulation of TRH gene expression in the paraventricular nucleus of the hypothalamus (PVH) by leptin is critical for normal function of the thyroid axis in rodents and humans. The TRH neuron in the PVH expresses both leptin and melanocortin-4 receptors, suggesting that both signaling systems may regulate TRH gene expression in vivo. Indeed, the TRH promoter responds to both of these signaling pathways in cell culture through identified cis-acting elements, which include signal transducer and activator of transcription (STAT) 3 and cAMP-response element binding protein-binding sites that mediate leptin and melanocortin responses, respectively.

Methods: The authors wanted to determine whether leptin signaling can directly target the TRH promoter in vivo. For this purpose they developed a chromatin immunoprecipitation assay to use on leptin-treated animals.

Results: After a single injection of leptin in fasting animals, the authors detected a significant increase in TRH gene expression in the PVH that correlated well with the induction of phosphorylated-STAT3 in the hypothalamus. In addition, it could be demonstrated that by using a STAT3 antibody the STATbinding site containing regions of both the TRH promoter and the promoter of the suppressor of cytokine signaling-3 gene could be immunoprecipitated, thereby underscoring the direct link to another well-defined target of leptin action. In contrast, upstream regions of these promoters that lack STAT sites were not precipitated.

Conclusions: The authors conclude that these experiments demonstrate that STAT3 mediates transcriptional effects of leptin in vivo and that the TRH promoter is a likely direct site of leptin action. In addition, these experiments demonstrate that chromatin immunoprecipitation can be used to characterize leptin signaling in vivo.

This is the description of a classic experiment using chromatin immunoprecipitation which was not so much geared towards defining a time- or dose-dependent response, but rather to prove a direct link between leptin levels in the PVH and TRH expression. This paper however is a nice description of the experimental approach of a ChIP experiment and how such an approach can be used in endocrinology to prove the specificity of an interaction that could not be deduced from simple hormone measurements or expression studies per se. 


\title{
Epigenetic mechanisms in the dopamine $\mathrm{d} 2$ receptor-dependent inhibition of the prolactin gene
}

\author{
Liu JC, Baker RE, Chow W, Sun CK, Elsholtz HP \\ Department of Laboratory Medicine and Pathobiology, University of Toronto, Toronto, Ont., Canada \\ Mol Endocrinol 2005; 19:1904
}

Background: Transcription of the prolactin gene is dynamically controlled by various positive and negative hormone signals that target the regulatory promoter region. Based on the inducibility of prolactin gene expression by inhibitors of histone deacetylases (HDACs) the authors examined the role of histone acetylation at the genomic prolactin promoter as a late step in transcriptional regulation.

Methods: The authors used chromatin immunoprecipitation analysis of GH4 cells to demonstrate elevated levels of acetylated histones in the promoter and enhancer regions of the gene, compared with downstream intron sequences.

Results: Whereas $17 \beta$-estradiol stimulated histone $\mathrm{H} 4$ acetylation in the promoter region 2- to 3-fold within $30 \mathrm{~min}$, dopamine inhibited histone $\mathrm{H} 4$ acetylation 2-fold in $30 \mathrm{~min}$. This effect could be mimicked by a MAPK kinase (MEK1) inhibitor U0126. In contrast, the synthetic glucocorticoid dexamethasone, which inhibits prolactin transcription, failed to alter histone acetylation over the same time frame. On the other hand the association of transcription activator Pit- 1 with the prolactin promoter was unchanged by hormone treatment. In response to dopamine however, histone deacetylase HDAC2 and corepressor $\mathrm{mSin} 3 \mathrm{~A}$ were rapidly recruited to the prolactin promoter, and association was sustained above basal levels over a 1-hour period. The authors found that in consistence with this corepressor function, depletion of endogenous $\mathrm{mSin} 3 \mathrm{~A}$ by small interfering RNA was sufficient to enhance prolactin gene expression by $70 \%$, comparable to the induction by the HDAC inhibitor, trichostatin A.

Conclusions: The authors conclude that dopamine D2 receptor activation and inhibition of MAPK (ERK1/2) signaling lead to rapid deacetylation of histones at the genomic prolactin promoter. They speculate that the recruitment of specific HDAC/corepressor complexes may be an important mechanism for repression of target gene transcription by Gi/o-coupled receptors.

This report demonstrates that the technique of chromatin immunoprecipitation (ChIP) enables timedependent studies on promoters on a different time scale as for example the photobleaching experiments described above. They seem more suitable to examine mechanisms with a longer turnover rate (several minutes) than observed for example in the glucocorticoid receptor (see above). A combination of both techniques might seem the appropriate approach. However there still remains a time window that may not be covered by any of these new techniques. This demonstrates that one has to be aware of the properties of the promoter itself and of the biochemical characteristics of its regulators to adequately use functional analyses that resolve time-dependent effects of nuclear regulators. 